\title{
INDUCTION OF BROWN ADIPOSE TISSUE: A REVIEW
}

\author{
IVO ROMAULD SAGAYARAJ*, AKILASHREE S, BRINDHA DEVI P
}

Department of Bio-Engineering, School of Engineering, VISTAS, Pallavaram, Chennai, Tamil Nadu, India. Email: ivoromauld@gmail.com Received: 18 September 2017, Revised and Accepted: 13 February 2018

\begin{abstract}
Objective: Obesity is the major problem which may lead to many other health ailments such as atherosclerosis, stroke, and depression. Both the cause as well as the treatment lies in the adipose tissue. The two main adipocytes, white adipose tissue (WAT) and brown adipose tissue (BAT) are responsible for the accumulation of fat and transformation of fat into heat, respectively. This review discusses the induction of BAT and browning of WAT by different pathways and activators to decrease the rate of obesity.
\end{abstract}

Methods: Understanding the regulators, activators and secreted proteins which induce browning of WAT to BAT, as the BAT engage in thermogenesis process and transform fat into heat rather than storing it (WAT). Some of the core regulators are peroxisome proliferator-activated receptor- $\gamma$, PRDM16, PGC-1 $\alpha$.

Results: A basic study explained about the origin of BAT and its functions, the function of hormones in BAT growth and its regulations. These studies provided the platform to understand about the mechanism of regulators, activators and secreted proteins which help in treating obesity and its related disorders by inducing the amount of BAT.

Conclusion: The major health ailments caused by obesity can be reduced by increasing the activity of BAT and transforming WAT into BAT. A challenging way to treat these ailments is by regulating the activators and hormones responsible for the induction of BAT, so it transforms the excess fat into heat and avoiding the accumulation of fat. By understanding the role of regulators in the adipose tissue can provide various methods to reduce the chance of obesity and enhance efficient treatment in both children and adults.

Keywords: Brown adipose tissue, White adipose tissue, uncoupling protein-1, Irisin hormone, Thermogenesis, peroxisome proliferator-activated receptor- $\gamma$, PRDM16, PGC-1 $\alpha$.

(C) 2018 The Authors. Published by Innovare Academic Sciences Pvt Ltd. This is an open access article under the CC BY license (http://creativecommons. org/licenses/by/4. 0/) DOI: http://dx.doi.org/10.22159/ajpcr.2018.v11i5.22646

\section{INTRODUCTION}

The highest threat to the adults as well as the children is obesity and overweight caused by the intake of high-calorie food and leading a deskbound way of life [1]. As the energy intake and energy expenditure are not allied with each other, the energy gets transformed and gets stored as fat. Obesity is a major risk factor for many other disorders including cardiovascular disease, stroke, hypertension, arthritis, type 2 diabetes, and various types of cancer [1]. A challenging way to treat this is by brown adipose tissue (BAT). There are two types of adipose tissue: White adipose tissue (WAT) and BAT $[2,3]$. The main function of WAT is to store the excess energy left after the energy expenditure, in the form of triacylglycerols (TAGs), this differs from every individual. Whereas, the BAT releases energy in the form of heat through uncoupling reaction with the help of Uncoupling protein-1 (UCP1) expression [4,7]. When total energy intake exceeds the total energy expenditure, the obesity develops [4]. As some theories of human obesity have postulated a thermogenic defect carried out by the BAT as a primary factor (James and Trayhurn, 1976), these theories have given rise to the speculation that a defect in BAT may be an important cause of obesity in human (James and Trayhurn, 1981). BAT constitutes about $1 \%$ of total body in human infants, which is about $30 \mathrm{~g}$ of BAT and the amount gets reduced as they grow [4]. The quantity and activity of BAT are correlated to the capability of humans to defy body fat buildup [6]. Recently, studies have shown that even adults have the BAT which can be stimulated when exposed to cold temperature; hence, BAT falls in the non-shivering thermogenesis process [5]. The non-shivering thermogenesis process protects the vital body parts from the extremely cold temperature [5]. The cold temperature stimulates the action of BAT and initiates the thermogenesis process with the help of the UCP1; This also induces the browning of WAT. Hence, these are the main factors responsible to convert the fat into heat. UCP1, which is present in the BAT and is responsible for the thermogenic activity. Hence, this reduces the accumulation of fat in the tissues and the thermogenesis process occurs to transform excess fat into heat. Other than the WAT and BAT, the beige adipocytes are also present. The regulators which are present as the specific gene can be stimulated to produce beige adipocytes which have both properties of brown and white adipocytes. Beige adipocytes are an inducible form of thermogenic adipocytes. Normally, it has low UCP1 content in basal conditions but when it gets induced with the help of regulators UCP1 level gets increased as in brown adipocytes [8]. In this review, we are going to look at the factors or regulators responsible for the browning of WAT-BAT, the formation of beige adipocytes (high UCP1 content).

\section{Understanding the function of adipose tissue and its types}

Earlier, adipose tissue was not considered as important as now. Recent studies have stated that the adipose tissue as an endocrine organ as it releases many complex products for the energy metabolism. Released products are adipokines such as leptin, adiponectin, and other cytokines, retinol binding protein-4 (RBP-4) and interleukin-6 (IL-6). This is also an important site for the metabolism of glucocorticoids and sex steroids $[9,10]$. The released products act on other tissues such as tissues in liver, brain, and muscle to regulate the fat accumulation, insulin sensitivity, energy metabolism, and reproduction $[9,10]$. There are two main types of fat found in the body; They are subcutaneous fat and visceral fat. The subcutaneous fat is found in the skin which is mainly functions to provide insulation and the other is the epididymal/ visceral fat, which is found in the organs (hard to remove). BAT is one of the two types of adipose tissue which involve in heat generation by a process called as thermogenesis. BAT mainly occurs within the thigh, back, abdomen, axillae, mediastinum, and neck [5]. The other type is 
WAT which is primarily a storage site which stores fat in the form of TAGs. BAT transforms the fat into heat by various factors and enhances leanness. The other type is the beige adipocytes which are also called as an inducible form of thermogenic adipocytes. Beige adipocytes do not have similar cell lineage with the BAT. Beige and white adipocytes develop from the WAT precursor cells with the inducement by $\beta 3$ adrenergic agonists [11]. BAT is present in newborns and other hibernating mammals in the higher amount. It appears brown in color due to the presence of mitochondria in it. This mitochondrion does not produce ATP due to the presence of UCP in their inner membrane. This BAT takes calorie from other normal fats and converts them into heat, thus leading to weight loss. UCP1 plays a major role in maintaining weight. Besides this, BAT is used as a potential source to treat diabetics $[12,13]$.

\section{Brown, white, and beige adipocytes}

As the name explains, BAT contains more mitochondria which do not involve in energy production rather uncouples the oxidative phosphorylation from ATP production $[7,8]$. They have multilocular fat droplets and an elevated level of UCP1 within the inner membrane of mitochondria [8]. The UCP1 which is present in the BAT release the electrons produced during the electron transport chain rather than storing for the energy production, this result in heat release. The factor influencing the brown adipocytes activity is norepinephrine [14,15]. The sympathetic nervous system involves the regulation of the thermogenic activity of BAT. Norepinephrine is released to activate the thermogenesis process [16]. This leads to the breakdown of triglycerides and discharge of free fatty acids which are the regulators of UCP1 expression and the substrates for thermogenesis [8]. This stimulates the activity of UCP1. When BAT gets activated it can release chemical energy stored as triglycerides by introducing fatty acids into $\beta$ oxidation [8]. UCP1 functions to uncouple electron transport from ATP production instead it produces heat through BAT [8].

The development of BAT initiates at the embryonic stage. The main BAT depots in human infants are present in the interscapular region, whereas it is absent in the adults. Majority of the brown cells originate from their own precursor cells in the embryonic mesoderm where these precursor cells briefly express Pax7 and Myf5, two genes that were previously misunderstood as the genes of the skeletal myogenic cells in the mesoderm [11]. When the relationship between muscle and BAT is noted, brown fat precursor cells express a muscle-like gene type $[4,6]$.

White adipocytes have unilocular fat droplets, few mitochondria compared to BAT and its function is to store fat. When considering the other type of adipose tissue, beige adipose tissue does not have the account of Myf5 expression (BAT), as this arises from the WAT, where WAT with large locules gets transformed into beige cells in response to cold or $\beta 3$-adrenergic agonists and WAT does not respond to these agonists [4]. The thermogenic activity gets induced in colder temperatures, and it is reversible that is losing their UCP1 expression when exposed to warmer climates $[4,7,8]$. The beige adipocytes require constant stimulation to regulate the thermogenic activity. The white and the beige adipocytes develop from the precursors called bipotent precursors (as they give rise to both type adipocytes) which express platelet-derived growth factor receptor- $\alpha$ (PDGFR- $\alpha$ ) which are intimately linked with blood vessels. When they are introduced with $\beta 3$ agonists, it loses the PDGFR- $\alpha$ expression and differentiates into UCP1 containing adipocytes. In converse, PDGFR- $\alpha$ differentiates into white adipocytes when the high-fat diet is introduced [11].

\section{Evolution of beige adipose tissue}

Brown adipocytes are positioned in their specific depots which express elevated levels of thermogenic genes, whereas the beige adipocytes develop in white fat in response to activator/inducers. The UCP1 expressing fat tissues also get expressed in WAT in response to various stimulations by regulators and other stimulators $[11,16]$. These adipocytes are called as, recruitable BAT, white adipose BAT, beige or induced BAT. A prominent difference between the two cell types is that brown adipocytes develop thermogenic genes and high levels of UCP1 even under unstimulated conditions, whereas beige adipocytes express these properties only in the presence of some activators such as peroxisome proliferator-activated receptor- $\gamma(\operatorname{PPAR}-\gamma)$ agonists of the $\beta$-adrenergic receptor and other regulators such as PRDM16, PGC- $1 \alpha$, and irisin hormone [11].

\section{Need for inducing the action of BAT}

The functions of BAT are to reduce the fat which causes obesity by mitochondrialuncouplingwasinitiallybeentriedasthetherapy for weight loss [11]. There are various factors affecting obesity such as heredity, food intake, lifestyle, environmental factors, and the risks in obesity are also very high such as hypertension, diabetes, cardiac alterations, cancer, and other neurological disorders [62]. Brown adipocytes consist of abundant mitochondria which contain UCP1. UCP lay in the class of mitochondrial anion carrier proteins. These UCP's split-up oxidative phosphorylation from ATP synthesis with the energy released as heat called the mitochondrial proton leak [11,19]. The uncoupler 2,4-dinitrophenol (DNP) helps the protons to pass across the mitochondrial membrane, which is same as the effect of activated UCP1. Before, DNP was used as an effectual cut down (diet) pill for obesity, which clearly provides the proof for the mitochondrial uncoupling as an approach for obesity. However, DNP led to the severe side effects when high doses are introduced to the body, which includes tachypnea, hyperthermia, diaphoresis, tachycardia, and even death [11,17]. Thus, the objective is to build up strategies that improve respiratory uncoupling, particularly in adipocytes by exploiting the processes that evolved naturally to do this in BAT and beige adipocytes [11]. BAT is a prominent thermogenesis site in mammals which is the target to enhance weight loss. The heat released by the process of thermogenesis through BAT is necessary for the hibernators for enduring the cold environments. The UCPs helps in the transmission of anions from the inner mitochondrial membrane to the outer and vice versa [11,18]. UCP1, when triggered, it bypasses the electrochemical gradient that drives ATP synthesis and therefore stimulates respiratory chain activity. Heat, which is being produced as the by-product, is from the combustion of accessible substrates and distributed all over the body by circulation $[11,18,19]$.

\section{Currently available antiobesity drugs}

Another targeted region for the obesity control is pancreatic lipase (PL) which be the vital enzyme secreted by the pancreas, responsible for the metabolism of fat. PL breaks down the fat molecules (triglycerides) into smaller substances (monoglycerides) for the absorption of fat in small intestines [56]. There are many antiobesity agents which have been discovered over decades, inhibit the PL activity to reduce the intestinal absorption of fat. Some examples of PL inhibitors are orlistat, fluoxetine, and sibutramine to reduce or control weight. Orlistat which is the natural PL inhibitor and has been isolated from bacterial species, Streptomyces toxytricini $[56,57]$. The effect of the drug also induces some side effects such as loose stool, fecal urgency, and increase in blood pressure. These drugs work on suppressing the amount of food intake by controlling the appetite and cause some side effects such as dry mouth and insomnia [57]. Resveratrol, a stilbenoid produced by the plant's works against the effects of obesity by activating the gene called SIRT1 gene and side effect include bleeding [58].

\section{DISCUSSION}

BAT thermogenesis takes place in its compactly packed mitochondria which contains the BAT-specific protein (UCP1). Norepinephrine activates a signaling flow that activates UCP-1, which then uncouples aerobic respiration by stimulating the mitochondrial proton leak, thus generating heat instead of ATP $[3,7]$. There are many signaling pathways, activators, and regulating factors which induce the formation of beige adipocytes/BAT [8].

\section{Novel factors and signaling pathway}

Important pathways and factors that can induce the formation of BAT and beige are fibroblast growth factors 21 (FGF21), retinaldehyde 
(Rald), cAMP-mediated PKA, and p38 MAPK signaling pathway. The other factors are the major classes such as bone morphogenetic proteins (BMPs) and cardiac natriuretic peptides (NPs) which are secreted to induce browning in WAT [8].

FGF21 overexpression leads to weight loss by increasing the energy expenditure irrespective of food intake and FGF21 be the important regulator in metabolic processes $[29,31,32]$. The fat targeted by FGF21 is epididymal WAT and induced when exposed to cold environments. Expose to cold environments also induces an increase in plasma FGF21 level [21]. FGF21 also stimulates the UCP1 gene expression in both WAT and BAT [32]. FGF21 is also considered as an endocrine factor secreted in BAT, muscle, and liver. Exposure to cold conditions releases FGF21 in BAT through p38 MAPK activation [30]. Lack of FGF21 will result in an inability to adapt to cold environments and browning of WAT is reduced [8].

The cardiac NPs and atrial NP are the key hormones in hemodynamic homeostasis which are expressed in adipocytes to increase the breakdown of lipids (lipolysis) [33]. The NPs promote browning of WAT and enhance thermogenic gene expression in BAT [34]. There are two classes of NPs, ANP, and BNP which activate UCP1 expression and PGC- $1 \alpha$ expression in WAT and BAT (causes of browning). NP binds to NP receptor A which sustain guanylyl cyclase activity to produce cyclic guanosine monophosphate (cGMP). This production leads to the activation of PKG (cGMP-dependent protein kinase), which works parallel to PKA pathway [34].

BMPs are the members of growth factor $\beta$ which play a major role in differentiation of adipocytes and energy expenditure [35]. BMP4 promotes differentiation in WAT and BMP7 regulates BAT differentiation and energy expenditure. BMP7 is required for promoting BAT quantity and elevation in UCP1 for thermogenesis process. Expression of BMP7 results in an increase in the amount of BAT accumulation, elevation in energy expenditure, and weight loss [36]. Expression of BMP4 in WAT results in WAT browning (regulated by PGC- $1 \alpha$ ) and promotes insulin sensitivity as there is an elevation in metabolic rate [37].

Retinaldehyde induces browning of WAT protecting from diabetes and obesity. Originally, retinaldehyde dehydrogenase 1 enzyme converts retinaldehyde to retinoic acid which is found in visceral fat (deep fat). This enzyme reduces the browning process and induces weight gain. Retinaldehyde promotes UCP1 expression and PGC- $1 \alpha$ [38].

\section{By core regulators}

PPARY

The treatment with PPAR $\gamma$ rosiglitazone activator induces the UCP1 expression, accountable for thermogenesis in WAT of both human and mouse. The activation of PPAR $\gamma$ induces beige adipocytes in mouse which is derived from white preadipocytes differentiated to mature adipocytes. PPAR $\gamma$ plays an important role in adipocyte transdifferentiation as it is the essential factor responsible for the survival of mature adipocytes [27]. As the recent immunocytochemical studies state that, the PPAR $\gamma$-derived mature adipocytes (WAT to BAT) are with the UCP1 expression in at least $10 \%$ of the cells [4]. These cells also have increased PGC-1 $\alpha$ expression and Hoxc9 (homeobox C9) which is a WAT-specific gene marker, not present in the standard brown adipocytes and the cells do not express BAT-specific transcription factors Meox (mesenchyme homeobox), Lhx8 (LIM homeobox 8), and Zic1 (zinc finger protein of the cerebellum 1) [4]. The main types of fat found are subcutaneous (beneath the skin) and visceral/epididymal fat (around the organ). Subcutaneous is highly prone to browning and epididymal fat is difficult to target and less susceptible to browning. PPAR $\gamma$ targets the visceral/epididymal WAT [4]. It also functions as the receptor for the class called thiazolidinediones; These drugs are employed for treating type 2 diabetes mellitus [22].

PPAR $\gamma$ gets activated by both synthetic and natural ligand binding such as 15-deoxy-12, 14-prostaglandin $\mathrm{J}_{2}$, 9- and 13-HODE, and linoleic acid [23-26]. PPAR $\gamma$ plays an important role in adipocyte transdifferentiation as it is the essential factor responsible for the continued existence of mature adipocytes [27]. The adipocytes which are PPAR $\gamma$ deficient die very soon which are replaced by the adipocytes expressing PPAR $\gamma$ derived from fibroblast-like preadipocytes [27]. The studies including the expression of PPAR $\gamma$ in adipocytes states that the PPAR $\gamma$ overexpression in fibroblasts causes adipogenesis and the SC cells lacking PPAR $\gamma$ cannot differentiate into adipocytes.

Recent studies prove that the ebf2 (early B cell factor-2) functions as the cofactor which regulates PPAR $\gamma$ binding activity to WAT to initiate browning. In in vivo conditions, the ebf2 is required for the BAT development $[4,20,55]$.

The browning process involves the BAT genes induction and WAT genes repression [4]. The other regulator of browning process is the PRDM16 which is involved in the development of brown fat, is associated with the PPAR $\gamma$ ligand binding, i.e., to induce browning process, the PPAR $\gamma$ ligands require full agonism, which is satisfied by the activation of the PRDM16 pathway [28]

\section{PRDM16}

PRDM16 is a protein and encoded by PRDM16 gene is essential and sufficient to encourage brown adipogenesis in WAT, which is the determinant factor of BAT development [39]. The specific role of PRDM16 is the WAT selective cofactor [4]. It is a zinc finger protein which induces the browning fat differentiation [43]. It is also required for the activity of PPAR $\gamma$ regulator. Unlike PPAR $\gamma$, browning occurs in subcutaneous WAT. Similar to PPAR $\gamma$, PRDM6 is involved in the induction of BAT genes and the repression of WAT genes [39]. PRDM16 functions by associating with the coactivators PGC- $1 \alpha$ and PGC- $1 \beta$ can suppress the other binding proteins like CtBPs to enhance the browning of WAT [42].

PRDM16 and PPAR $\gamma$ are correlated with each other. PRDM16 functions with PPAR $\gamma$ to initiate the browning in WAT. As the visceral WAT are less prone to browning [43]. When the PRDM16 is overexpressed, the browning of primary visceral preadipocytes gets transformed into mature adipocytes $[4,28]$. Cells switch from myoblasts to BAT cells which are controlled by the action of PRDM16 by forming a transcriptional complex with C/EBP- $\beta$ (which contains activation domains and regulatory domains) [43].

Expression of PRDM16 controls the binding of PPAR ligands with the subcutaneous WAT. These also determine the browning of WAT [28]. The effects of browning in subcutaneous WAT can be seen with the increase of energy expenditure and elevated glucose tolerance level $[39,40]$.

\section{PGC-1 $\alpha$}

PGC- $1 \alpha$ is also correlated with PRDM 16. The expression of PGC- $1 \alpha$ gets elevated during the cold exposure. This plays a major role in adaptive thermogenesis by linking nuclear receptors to the transcription process of the adipocytes [44]. The recent studies stated in subcutaneous WAT, expression of PGC- $1 \alpha$ leads to the amplified expression of UCP1, fatty acid oxidation enzymes, CIDEA mRNA, and respiratory chain proteins [4]. The expression of PGC- $1 \alpha$ is required for inducing UCP1 and other BAT-specific genes to enhance browning process in WAT [45]. The browning does not occur with the help of single regulator, the PGC-1 $\alpha$ with muscle-secreted myokine (irisin) engage in the browning process of WAT. The expression of PGC- $1 \alpha$ stimulates the secretion of irisin hormone which is derived from fibronectin-type 3 domain-containing 5 (FNDC5) (membrane protein) [46]. The browning process is proved by the immunohistochemistry study which reveals that the presence of multilocular cells with UCP-1 positive is a sign of browning [46].

The protein called $\mathrm{pRb}$ (retinoblastoma protein) and $\mathrm{Rb}$ family member p107 acts as the coregulator for the expression of PGC- $1 \alpha$ [47]. However, the recent study proved that these regulators have turned into negative 
regulators and repressed the PGC- $1 \alpha$ expression. In contrast, FGF21 enhances the PGC- $1 \alpha$ expression and be a positive regulator which enhances the browning process [32].

\section{Browning of WAT by irisin (exercise)}

Irisin, a recently identified hormone encoded by the gene FNDC5, which is secreted by the skeletal muscles and lies in the category of musclesecreted myokine, promotes browning of WAT [4]. PGC-1 $\alpha$ with musclesecreted myokine (Irisin) engages in the browning process of WAT. The expression of PGC- $1 \alpha$ stimulates the secretion of irisin hormone which is derived from FNDC5 (membrane protein) [46]. By the mitogen-activated protein kinase P38 (MAPK) and ERK MAPK signaling, irisin stimulates the WAT browning [6]. It reduces the diet-induced obesity as it mainly concentrates on WAT browning and mediates the useful effects of exercises [6]. This also targets the subcutaneous WAT. Irisin is a myokine which is induced by physical activities (exercise) and secreted into the circulation by the proteolytic cleavage of its cellular form [21].

Irisin stimulates thermogenesis process in rodents through increasing the beige adipocytes in WAT. This reduces the effect of diet-induced obesity and diabetes $[4,6]$. Exercise increases the levels of serum irisin in humans. Shivering of the body is the signal of irisin secretion [21]. The recent study provided sufficient information about the effect of irisin hormone in weight loss therapies, i.e., it decreases the body weight and enhances the glucose homeostasis [6]. Also, the Irisin hormone upregulated the expression of UCP-1. This process is possibly mediated by ERK signaling pathways and irisin-induced phosphorylation of the P38 MAPK [6]. In addition to the enhancement of thermogenesis process, irisin also promotes the betatrophin expression, a hormone which improves the glucose tolerance and pancreatic $\beta$-cell proliferation [6].

\section{Cold exposure}

The longtime exposure to cold environment stimulates the action of BAT and initiates the browning of WAT. The coldness is sensed by the thermoreceptors (specialized nerve cells that are able to detect the difference in temperature) $[8,48]$. Cold condition acts as a stimulus to produce beige fat. BAT sympathetic premotor neurons in the rostral ventromedial medulla are activated by thermosensory neurons sensing to the cold exposure. This leads to the BAT thermogenesis [48].

As a result, the norepinephrine is released, which activates the action of BAT by releasing the inducers of the UCP1. Recent studies have elucidated that the catecholamine and tyrosine hydroxylase are the factors responsible for the browning of subcutaneous WAT when exposed to cold environments [8]. The beige fat (eosinophils) consisting of type 2 cytokines IL, alternatively activated macrophages and the eosinophils regulate the cold-induced beige fat development [49]. Introduction of type 2 cytokines IL- $4 / 3$ leads to the increase in the beige fat amount and enhances its thermogenic property [49]. The regulation of the beige fat development leads to the production of catecholamine and expression of tyrosine hydroxylase; these expressions lead to the subcutaneous WAT browning [8]. For long period adaptations to cold environments, meteorin-like (Metrnl) is a hormone which gets secreted on the cold environment and stimulates the browning process by inducing M2 macrophage activation and IL-4/13 $[8,50]$. The secretion of Metrnl leads to the enhancement of glucose tolerance level and increases in energy expenditure and expression of BAT-specific genes [50].

\section{Exercise}

When the exercise is considered, it deals with the body metabolism (glucose, lipids metabolism, and energy expenditure). The studies have stated that it increases the browning of WAT and elevates the energy expenditure. As the skeletal muscles and adipocytes are interrelated with each other, PGC- $1 \alpha$ is induced in the muscle which leads to browning process [46]. IL-6 is the myokine produced by exercise with exposure to very cold conditions. Expression of UCP1 is enhanced which play a major role in the browning of WAT [52].
As PGC- $1 \alpha$ is correlated with the secretion of irisin hormone, the browning process by the hormone is also activated by transforming the membrane protein FNDC5 into irisin [51], and Metrnl (myokine) is induced by the PGC- $1 \alpha 4$ which promotes browning of WAT [50]

\section{Treatment for obesity and its related disorders}

Triglycerides are targeted by the BAT which plays a major role in obesity conditions and cardiovascular conditions. BAT activity can trigger plasma clearance of triglycerides by exposing to cold conditions. Increase in the activity of BAT and WAT browning leads to increase in energy expenditure. BAT thermogenesis leads to improved insulin resistance and corrected hyperlipidemia. BAT can also clear up the triglycerides from the circulation [53].

BAT transplantation can be done to the visceral regions to induce weight loss and improves glucose tolerance level. Increase in amount of BAT to be transplanted varies according to the metabolic conditions. Reversal of HFD-induced insulin resistance is achieved by the BAT transplantation. IL- 6 is required to enhance the effects of BAT transplantation [54]. Thus, these specific cell types are the therapeutic target for obesity and related disorders.

\section{CONCLUSION}

Obesity in children provides a unique set of challenges, leading to major health ailments. The cause and the treatment lie in the same surrounding. As the cause of obesity and their related ailments lies in the WAT and the treatment for the ailments is in the BAT. BAT which transforms fat into heat and avoids accumulation of fat, occurs to be an attractive therapeutic target for controlling obesity. By the process called adaptive thermogenesis, BAT regulates the energy expenditure where the chemical energy is converted to heat. Adipocytes open a diverse range of therapeutic interventions, hormones, regulators, and inducers which could induce browning process. This process reduces the complications of obesity and its related problems. There are some drawbacks to overcome to obtain the maximum efficiency of the different therapies. There are some depot differences found between subcutaneous WAT and visceral WAT, as the subcutaneous is more prone to browning process than the epididymal WAT, the cause for the major health ailments in the body. Epididymal WAT has to be reduced by the specific regulators targeting specific WAT. However, there are many processes and concepts which are a bit confusing, like whether browning is a result of transdifferentiation of WAT or the beige adipocytes possess inherent characteristics to browning process. These are the future directions in the field of clinical cell biology to understand the adipocytes morphology and functions. If these drawbacks are overcome by further studies, the stimulation of BAT is the successful treatment for obesity and obesity-related disorders.

\section{REFERENCES}

1. Seale P, Lazar MA. Brown fat in humans: Turning up the heat on obesity. Diabetes 2009;58:1482-4.

2. Enerback S. The origins of brown adipose tissue. N Engl J Med 2009;360:2021-3.

3. Cypess AM, Kahn CR. The role and importance of brown adipose tissue in energy homeostasis. Curr Opin Pediatr 2010;22:478-84.

4. Lo KA, Sun L. Turning WAT into BAT: Areview on regulators controlling the browning of white adipocytes. Biosci Rep 2013;33:e00065.

5. Carter BW, Schucany WG. Brown adipose tissue in a newborn. Proc (Bayl Univ Med Cent) 2008;21:328.

6. Zhang Y, Li R, Meng Y, Li S, Donelan W, Zhao Y, et al. Irisin stimulates browning of white adipocytes through mitogen-activated protein kinase p38 MAP kinase and ERK MAP kinase signaling. Diabetes 2014;63:514-25

7. Sidossis L, Kajimura S. Brown and beige fat in humans: Thermogenic adipocytes that control energy and glucose homeostasis. J Clin Invest 2015;125:478-86

8. Kim SH, Plutzky J. Brown fat and browning for the treatment of obesity and related metabolic disorders. Diabetes Metab J 2016;40:12-21.

9. Jung UJ, Choi MS. Obesity and its metabolic complications: The role of 
adipokines and the relationship between obesity, inflammation, insulin resistance, dyslipidemia and nonalcoholic fatty liver disease. Int J Mol Sci 2014; 15:6184-223.

10. Kershaw EE, Flier JS. Adipose tissue as an endocrine organ. J Clin Endocrinol Metab 2004;89:2548-56.

11. Harms M, Seale P. Brown and beige fat: Development, function and therapeutic potential. Nat Med 2013;19:1252-63.

12. Blaza S. Brown adipose tissue in man: A review. J R Soc Med 1983;76:213-6.

13. Ricquier D, Bouillaud F. Mitochondrial uncoupling proteins: From mitochondria to the regulation of energy balance. J Physiol 2000;529:3- 10 .

14. Inokuma K, Ogura-Okamatsu Y, Toda C, Kimura K, Yamashita H, Saito $\mathrm{M}$, et al. Uncoupling protein 1 is necessary for norepinephrineinduced glucose utilization in brown adipose tissue. Diabetes 2005;54:1385-91.

15. Cannon B, Nedergaard JA. Brown adipose tissue: Function and physiological significance. Physiol Rev 2004;84:277-359.

16. Kajimura S, Spiegelman BM, Seale P. Brown and beige fat: Physiological roles beyond heat generation. Cell Metab 2015;22:546- 59.

17. Grundlingh J, Dargan PI, El-Zanfaly M, Wood DM 2,4-dinitrophenol (DNP): A weight loss agent with significant acute toxicity and risk of death. J Med Toxicol 2011;7:205-12.

18. Brondani LA, Assmann TS, Duarte GC, Gross JL, Canani LH, Crispim D, et al. The role of the uncoupling protein 1 (UCP1) on the development of obesity and Type 2 diabetes mellitus. Arq Bras Endocrinol Metabol 2012;56:215-25.

19. Mookerjee SA, Divakaruni AS, Jastroch M, Brand MD. Mitochondrial uncoupling and lifespan. Mech Ageing Dev 2010;131:463-72.

20. Gurnell M. PPAR $\gamma$ and metabolism: Insights from the study of human genetic variants. Clin Endocrinol 2003;59:267-77.

21. Lee P, Linderman JD, Smith S, Brychta RJ, Wang J, Idelson C, et al. Irisin and FGF21 are cold-induced endocrine activators of brown fat function in humans. Cell Metab 2014;19:302-9.

22. Rosen ED, Sarraf P, Troy AE, Bradwin G, Moore K, Milstone DS, et al. PPAR $\gamma$ is required for the differentiation of adipose tissue in vivo and in vitro. Mol Cell 1999;4:611-7.

23. Forman BM, Tontonoz P, Chen J, Brun RP, Spiegelman BM, Evans RM. 15-deoxy- $\Delta 12$, 14-prostaglandin $\mathrm{J} 2$ is a ligand for the adipocyte determination factor PPAR $\gamma$. Cell 1995;83:803-12.

24. Kliewer SA, Lenhard JM, Willson TM, Patel I, Morris DC, Lehmann JM. A prostaglandin $\mathrm{J} 2$ metabolite binds peroxisome proliferator-activated receptor $\gamma$ and promotes adipocyte differentiation. Cell 1995;83:813-9.

25. Kliewer SA, Sundseth SS, Jones SA, Brown PJ, Wisely GB, Koble CS, et al. Fatty acids and eicosanoids regulate gene expression through direct interactions with peroxisome proliferator-activated receptors $\alpha$ and $\gamma$. Proc Natl Acad Sci 1997;94:4318-23.

26. Nagy L, Tontonoz P, Alvarez JG, Chen H, Evans RM. Oxidized LDL regulates macrophage gene expression through ligand activation of PPAR $\gamma$. Cell 1998;93:229-40.

27. Imai T, Takakuwa R, Marchand S, Dentz E, Bornert JM, Messaddeq N, et al. Peroxisome proliferator-activated receptor $\gamma$ is required in mature white and brown adipocytes for their survival in the mouse. Proc Natl Acad Sci U S A 2004;101:4543-7.

28. Ohno H, Shinoda K, Spiegelman BM, Kajimura S. PPAR $\gamma$ agonists induce a white-to-brown fat conversion through stabilization of PRDM16 protein. Cell Metab 2012;15:395-404

29. Chartoumpekis DV, Habeos IG, Ziros PG, Psyrogiannis AI, Kyriazopoulou VE, Papavassiliou AG, et al. Brown adipose tissue responds to cold and adrenergic stimulation by induction of FGF21. Mol Med 2011;17:736-40.

30. Hondares E, Iglesias R, Giralt A, Gonzalez FJ, Giralt M, Mampel T, et al. Thermogenic activation induces FGF21 expression and release in brown adipose tissue. J Biol Chem 2011;286:12983-90.

31. Coskun T, Bina HA, Schneider MA, Dunbar JD, Hu CC, Chen Y, et al. Fibroblast growth factor 21 corrects obesity in mice. Endocrinology 2008;149:6018-27.

32. Fisher FM, Kleiner S, Douris N, Fox EC, Mepani RJ, Verdeguer F, et al. FGF21 regulates PGC-1 $\alpha$ and browning of white adipose tissues in adaptive thermogenesis. Genes Dev 2012;26:271-81.

33. Sengenès C, Berlan M, De Glisezinski I, Lafontan M, Galitzky J. Natriuretic peptides: A new lipolytic pathway in human adipocytes. FASEB J 2000;14:1345-51.

34. Bordicchia M, Liu D, Amri EZ, Ailhaud G, Dessì-Fulgheri P, Zhang C, et al. Cardiac natriuretic peptides act via p38 MAPK to induce the brown fat thermogenic program in mouse and human adipocytes. J Clin Invest 2012;122:1022-36.

35. Zamani N, Brown CW. Emerging roles for the transforming growth factor- $\beta$ superfamily in regulating adiposity and energy expenditure. Endocr Rev 2010;32:387-403.

36. Tseng YH, Kokkotou E, Schulz TJ, Huang TL, Winnay JN, Taniguchi CM, et al. New role of bone morphogenetic protein 7 in brown adipogenesis and energy expenditure. Nature 2008;454:1000-4.

37. Qian SW, Tang Y, Li X, Liu Y, Zhang YY, Huang HY, et al. BMP4mediated brown fat-like changes in white adipose tissue alter glucose and energy homeostasis. Proc Natl Acad Sci U S A 2013;110:E798-807.

38. Kiefer FW, Vernochet C, O'Brien P, Spoerl S, Brown JD, Nallamshetty S, et al. Retinaldehyde dehydrogenase 1 regulates a thermogenic program in white adipose tissue. Nat Med 2012;18:918-25.

39. Seale P, Bjork B, Yang W, Kajimura S, Chin S, Kuang S, et al. PRDM16 controls a brown fat/skeletal muscle switch. Nature 2008;454:961-7.

40. Kajimura S, Seale P, Kubota K, Lunsford E, Frangioni JV, Gygi SP, Spiegelman BM. Initiation of myoblast to brown fat switch by a PRDM16-C/EBP- $\beta$ transcriptional complex. Nature 2009;460:1154-8.

41. Seale P, Conroe HM, Estall J, Kajimura S, Frontini A, Ishibashi J, et al. Prdm16 determines the thermogenic program of subcutaneous white adipose tissue in mice. J Clin Invest 2011;121:96-105.

42. Kajimura S, Seale P, Tomaru T, Erdjument-Bromage H, Cooper MP, Ruas JL, et al. Regulation of the brown and white fat gene programs through a PRDM16/CtBP transcriptional complex. Genes Dev 2008;22:1397-409

43. Waldén TB, Hansen IR, Timmons JA, Cannon B, Nedergaard J. Recruited vs. Nonrecruited molecular signatures of brown,"brite," and white adipose tissues. Am J Physiol Endocrinol Metab 2012;302:E19- 31

44. Puigserver P, Wu Z, Park CW, Graves R, Wright M, Spiegelman BM, et al. A cold-inducible coactivator of nuclear receptors linked to adaptive thermogenesis. Cell 1998;92:829-39.

45. Pardo R, Enguix N, Lasheras J, Feliu JE, Kralli A, Villena JA. Rosiglitazone-induced mitochondrial biogenesis in white adipose tissue is independent of peroxisome proliferator-activated receptor $\gamma$ coactivator-1 $\alpha$. PLoS One 2011;6:e26989.

46. Boström P, Wu J, Jedrychowski MP, Korde A, Ye L, Lo JC, et al. A PGC1-[agr]-dependent myokine that drives brown-fat-like development of white fat and thermogenesis. Nature 2012;481:463-8.

47. Scimè A, Grenier G, Huh MS, Gillespie MA, Bevilacqua L, Harper ME, et al. $\mathrm{Rb}$ and $\mathrm{p} 107$ regulate preadipocyte differentiation into white versus brown fat through repression of PGC-1 $\alpha$. Cell Metab 2005;2:283-95.

48. Morrison SF, Madden CJ, Tupone D. Central control of brown adipose tissue thermogenesis. Front Endocrinol (Lausanne) 2012;3.

49. Qiu Y, Nguyen KD, Odegaard JI, Cui X, Tian X, Locksley RM, et al. Eosinophils and Type 2 cytokine signaling in macrophages orchestrate development of functional beige fat. Cell 2014;157:1292-308.

50. Rao RR, Long JZ, White JP, Svensson KJ, Lou J, Lokurkar I, et al. Meteorin-like is a hormone that regulates immune-adipose interactions to increase beige fat thermogenesis. Cell 2014;157:1279-91.

51. Handschin C, Spiegelman BM. The role of exercise and PGC1 $\alpha$ in inflammation and chronic disease. Nature 2008;454:463-9.

52. Knudsen JG, Murholm M, Carey AL, Biensø RS, Basse AL, Allen TL, et al. Role of IL-6 in exercise training- and cold-induced UCP1 expression in subcutaneous white adipose tissue. PLoS One 2014;9:e84910.

53. Bartelt A, Bruns OT, Reimer R, Hohenberg H, Ittrich H, Peldschus K, et al. Brown adipose tissue activity controls triglyceride clearance. Nat Med 2011;17:200-5

54. Stanford KI, Middelbeek RJ, Townsend KL, An D, Nygaard EB, Hitchcox KM, et al. Brown adipose tissue regulates glucose homeostasis and insulin sensitivity. J Clin Invest 2013;123:215-23

55. Rajakumari $\mathrm{S}, \mathrm{Wu} \mathrm{J}$, Ishibashi J, Lim HW, Giang AH, Won KJ, et al. EBF2 determines and maintains brown adipocyte identity. Cell Metab 2013;17:562-74

56. Sarkar SJ, Dioundi D, Gupta M. Endophytic Pestalotiopsis species from Andaman Islands: A potential pancreatic lipase inhibitor. Asian J Pharm Clin Res 2017;10:82-3.

57. Bollapragada M, Shantaram M, Sunil Kumar R. Obesity: Development, epidemiology, factors affecting, quantity, health hazards, management and natural treatment-a review. Int J Pharm Pharm Sci 2017;9:12-26.

58. Mohamed HO, Asker ME, Amin RA, Eissa RA. Anti-obesity effect of resveratrol in rats on high fat diet through regulation of gene expression of visceral white adipose tissue. Int J Pharm Pharm Sci 2016;8:378-84. 